\title{
Seroepidemiology of measles in Addis Ababa, Ethiopia: implications for control through vaccination
}

\author{
F. ENQUSELASSIE ${ }^{1}$, W. AYELE ${ }^{2}$, A. DEJENE ${ }^{3}, \mathrm{~T}_{\text {MESSELE }}^{4}, \mathrm{~A} . \mathrm{ABEBE}^{2}$, \\ F. T. CUTTS ${ }^{5}$ AND D. J. NOKES ${ }^{6 *}$ \\ ${ }^{1}$ Department of Community Health, Faculty of Medicine, University of Addis Ababa, PO Box 1176, \\ Addis Ababa, Ethiopia \\ ${ }^{2}$ Virology and Rickettsiology Research Team, Ethiopian Health and Nutrition Research Institute, \\ PO Box 1242, Addis Ababa, Ethiopia \\ ${ }^{3}$ Biostatistics and Health Service Research Team, Ethiopian Health and Nutrition Research Institute, \\ PO Box 1242, Addis Ababa, Ethiopia \\ ${ }^{4}$ Immuno-Haematology and Pathology Research Team, Ethiopian Health and Nutrition Research Institute, \\ PO Box 1242, Addis Ababa, Ethiopia \\ ${ }^{5}$ Department of Infectious and Tropical Diseases, London School of Hygiene and Tropical Medicine, \\ Keppel Street, London, WC1E $7 H T, U K$ \\ ${ }^{6}$ Department of Biological Sciences, University of Warwick, Coventry, CV4 7AL, UK
}

(Accepted 9 January 2003)

\section{SUMMARY}

We undertook a representative survey of measles antibodies in Addis Ababa, Ethiopia 1994, to characterize immunity and transmission. Specific-antibody levels (IU/1) were determined by ELISA for 4654 sera from individuals aged $0-49$ years $(1805<15$ years $)$ collected by stratified household-cluster sampling. The proportion seronegative ( < $100 \mathrm{IU} / \mathrm{l})$ was $20 \%(95 \% \mathrm{CI}: 16-25)$ in children 9-59 months old, declining to 9\% (7-12) in 5-9 year olds, 5\% (4-7) in 10-14 year olds, and $<1 \%$ in adults. The proportion of children ( $<15$ years old) with low-level antibody (100-255 IU/1) was $8 \%$ (7-10). Vaccination and an absence of a history of measles illness were strongly associated with low-level antibody. History of measles vaccination in 9 months to 14 -year-old children was $\sim 80 \%$. We estimate a primary vaccine failure rate of $21 \%(12-34)$ and continued high measles incidence of 22 per 100 susceptibles (19-24) per annum. Our data support the introduction of campaign vaccination in the city in 1998, although higher routine vaccine coverage is required to sustain the impact. The implications of a high prevalence of low-level antibody are discussed.

\section{INTRODUCTION}

Serological surveys of specific antibody status in vaccinated populations provide valuable information to health authorities on the impact of the intervention on continued transmission [1, 2], the distribution (by age and geographical location) of proportions

\footnotetext{
* Author for correspondence: Welcome Trust Research Labs, Kenya Units, PO Box 230, Kilifi, Kenya.
}

seronegative [3-5], the risks of an outbreak [1, 6], and the progress towards elimination [7]. Surveys that report on the level of detectable antibody, rather than presence or absence, may further inform on the likely distribution of sub-clinical, mild and typical measles re-infection and on the potential for community persistence of measles [3, 4, 8-10].

There is little recent data on measles seroepidemiology and the impact of vaccination on measles transmission in Ethiopia [11,12] or elsewhere in much 
of Africa. Here we report on a large representative survey of measles specific antibodies in Addis Ababa, in 1994, following over a decade of moderately high coverage of routine measles vaccination $(\sim 80 \%)$ and prior to the implementation of measles immunization campaigns. The aim of the study was to characterize in detail measles specific-immunity and transmission within a vaccinated urban developing country population, and assess the implications of the results for measles control by vaccination.

\section{METHODS}

\section{Survey details}

Emphasis is placed on details not presented in previous related publications [13-15]. Ethical approval was obtained from authorities in Ethiopia (Ethical Committee of the Ethiopian Health and Nutrition Research Institute) and the United Kingdom (St Mary's Research Ethics Committee, London University).

\section{Setting}

Addis Ababa is the capital city of Ethiopia situated on a high plateau (altitude approximately $2000 \mathrm{~m}$ ), with an urban population of $2.1 \mathrm{~m} \mathrm{[16]} \mathrm{and} \mathrm{adminis-}$ tratively organized into urban dwellers associations or kebeles (each of roughly 1500-2000 registered households [16]). The expanded programme on immunization (EPI) was introduced in Ethiopia in 1980, targeting children under 2 years of age, then from 1986 under 1 year [17], with a single measles dose at the World Health Organization's recommended age of 9 months. Data on measles vaccination coverage in Addis Ababa is unavailable for the 1980s, and official figures between 1992/3 and 1993/4 give the proportion under 1 year of age vaccinated at around $75-80 \%$ (Addis Ababa City Administration Health Bureau, personal communication). A measles campaign targeting children aged 9-59 months was conducted in nine cities in Ethiopia, including Addis Ababa, in November 1998, as part of the accelerated measles control strategy in Ethiopia [18].

\section{Survey design and implementation}

A stratified cluster-sampling survey design was adopted in which 35 households (15\% non-compliance assumed) were selected at random (from official registers) within each of 20 kebeles in the inner city (high density stratum) and 20 kebeles in the outer city, selected with probability proportional to estimated size. Sample size calculations, using standard methods and an assumed cluster sampling design effect of 2 [19], determined a minimum requirement of 150 samples for each age class (defined as single years up to age 4 years, and 5 year classes from 5-9 to 45-49 years) to provide acceptable precision of prevalence estimates, ie $95 \%$ confidence intervals (CI) of $\pm 7-12 \%$.

Households were visited between end of May and early October of 1994. A letter from the Health Bureau of Region 14 (Addis Ababa) was provided to officials of each selected kebele and to each household head informing of the nature of the study. If consent was given an interview was carried out with the head of the household by trained interviewers, eliciting detail on household socio-demography and socioeconomics. Additionally, information was obtained on measles vaccine history for children $<15$ years of age [categorized as (i) positive card record of vaccination, (ii) positive history without card, (iii) negative card record or else, respondent negative, or (iv) respondent does not know] and past measles [categorized as (i) yes (ii) no or (iii) unknown] for all ages based upon recognition of classical symptoms. A blood sample was requested from all children under age 5 years, and adults aged 15-49 years, and from 1 in 2 of children aged 5-14 years by random selection. Up to two revisits were made if household members were previously unavailable. Sera were stored at $-20{ }^{\circ} \mathrm{C}$.

\section{Screening methods}

Serum samples were screened by a commercial EIA kit for measles-specific IgG antibodies (Rubeola G, Gull Laboratories, Atlas Bioscan Ltd, Bognor Regis, UK) [21] following the manufacturer's instructions, with calibration using a measles international standard allowing quantification of results in $\mathrm{IU} / 1[4,21]$.

\section{Data processing and analysis}

Data was double entered onto Epi-Info [22], with internal range checks, and later compared for consistency checking and cleaned.

\section{Classification of measles antibody status}

Based on the frequency distribution of (log) antibody levels in unvaccinated individuals, we defined as seronegative individuals whose measles-specific $\mathrm{IgG}$ 
antibody level were less than $100 \mathrm{IU} / 1$. Seropositive individuals were stratified by cut-off levels of 255 [4], 500 [7] and 1000 IU/1 [9].

\section{Statistical analysis}

Data were analysed using the statistical software STATA (Statacorp, v. 6.0, 1999, College Station, TX, USA) applying specific survey procedures ('svy' commands) that account for survey design. The kebele was defined as the primary sampling unit, and probability weights were applied to account for unequal cluster and stratum population sizes (details of which are described elsewhere $[13,15])$. Within these survey procedures, hypothesis testing uses the adjusted Wald test and Pearson's design-based $\chi^{2}$ test. Logistic regression (STATA svylogit command) was used to identify independent risk factors associated with measles seronegative status or with low seropositive status $(100-255 \mathrm{IU} / \mathrm{l})$. Variables identified as significant $(P<0.05)$ in univariate analysis were included in a multivariate model, followed by a stepdown procedure to remove those factors not contributing significantly $(P<0 \cdot 05)$.

\section{Estimation of measles incidence}

Seroprevalence data were analysed with the aim of estimating the force of infection acting on susceptibles (the per susceptible incidence rate) in a population with a vaccination programme in place. It is assumed that the proportion susceptible to infection at age $a, x(a)$, is accurately defined by the proportion seronegative in the serological survey. Analysis is confined to the age group below 15 years for which vaccination data was collected, and above which there is little observable change in seroprevalence. The estimation procedure makes the key simplifying assumption that the effect on seroprevalence of measles vaccination has been constant over the time-span of interest (1980-94), which appears reasonable from coverage data from official Addis Ababa sources and this survey. Changes, with age, in the proportion seronegative are assumed to be the result of measles transmission, the rate of which potentially varies both by age (differences in social mixing) and through time (as vaccination acts to reduce the rate of transmission). These factors are investigated. Ageprevalence of seronegativity may be affected by migration into the city, which we control for in the analysis. The method makes the further simplifying assumptions that all individuals seroconvert upon primary infection, measles associated mortality is negligible, and positive seroprevalence is not diminished by waning of antibody (whether vaccine- or wild-type induced) in the age group of interest $(<15$ years).

On this basis we estimated the force of measles infection using a piece-wise constant (PWC) catalytic infection model [13, 23] using year group data for ages 1-14 years. The force of infection is assumed constant within an age class $i, \lambda_{i}$, but can vary between age classes $i=1, m$, hence the proportion susceptible predicted by the model, $x^{\prime}(\mathrm{a})$

$x^{\prime}(a)=x^{\prime}\left(a_{i-1}\right) \exp \left[-\lambda_{i}\left(a-a_{i-1}\right)\right]$,

where $i$ is such that $a_{i-1} \leqslant a \leqslant a_{i}$, and $\lambda_{i} \geqslant 0$. The assumption is of an exponential decay in the proportion susceptible between age $a_{i-1}$ and $a$ due to an age-band specific constant force of infection, $\lambda_{i}$. We avoid explicitly defining the rate of loss of maternal antibody and the effect of vaccination in the first year of life by setting an initial condition for the proportion susceptible at age $0, x^{\prime}(0) . x^{\prime}(0)$ and $\lambda_{i}, i=l, m$, were parameters to be estimated from the data, which we did by maximum likelihood, and estimated $95 \%$ confidence limits assuming an approximate $\chi^{2}$ distribution of the log-likelihoods [24]. The constrained model assumes a constant force of infection over all ages (1-14 years). We explored a reduction in the age range used for parameter estimation (i.e. upper age of 10 or 5 years). To determine the presence of time (or age) dependent variation in the force of infection, the model fit using 2 or 3 age bands (1-5, 6-10 and $11-14$ years, or combination therein) was assessed using the likelihood ratio test [25].

\section{RESULTS}

\section{Survey population characteristics}

Individuals numbering 8638 from 1384 households were registered in the study, $53 \%$ in the inner city, with $7 \cdot 2 \%$ aged under 5 years, $32.5 \%$ under 15 years and $10 \cdot 2 \%$ aged $50+$ years. The age distribution closely reflected that reported in the 1994 census of Addis Ababa: $8.0 \%<5$ years, $31.6 \%<15,8.5 \% 50+$ (tabulated details are given elsewhere $[15,16]$ ), and shows that the per cent less than 5 years of age was significantly less than in $1984(12 \cdot 4 \%)$ [20]. The overall male:female ratio was $46 \cdot 3: 53 \cdot 7 \%$ with relatively more females than in the 1994 census $(48 \cdot 4: 51 \cdot 6 \%)$. For other characteristics for which comparison was 


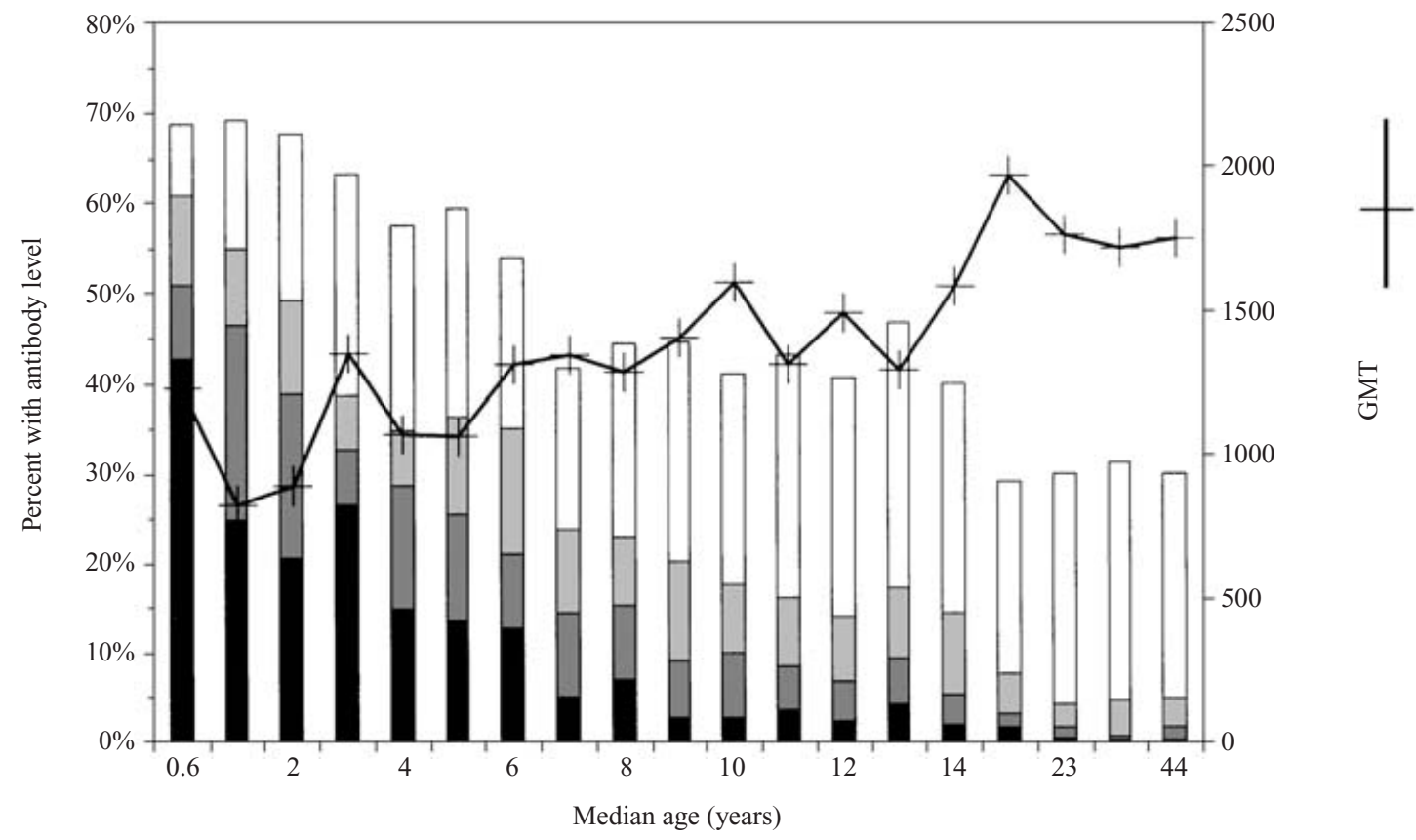

Fig. 1. Age-distribution of measles-specific antibody levels in Addis Ababa, 1994. The proportions (\%) seronegative (antibody levels < $100 \mathrm{IU} / 1$, black bars), with low-level antibody (100-255 IU/1, dark grey bars), or with levels of 255-500 IU/1 (light grey), or 500-1000 IU/1 (open bars), are shown for each age class. Geometric mean titre (GMT) of measles antibody (seropositives only) is shown by the continuous line (measured on the right vertical axis). The median age of classes $0-11$ months, $1,2, \ldots 14$ years, 15-19, and 20-29 to 40-49 years are shown and have the corresponding sample sizes: 55, 69, 69, $100,124,108,90,141,125,145,175,114,178,155,157,890,985,599,375$ (total 4654).

possible, we found close agreement with the census: average household size (de jure population) $5 \cdot 3$ (census $5 \cdot 5$ ), non-migrants (resident $\geqslant 10$ years) $87 \%$ (census $80 \%$ ), literacy level $83 \%$ (census $83 \%$ ), and ethnicity, $49 \%$ Amharas (49\%), 17\% Oromos $(19 \%), 9 \%$ Tigre $(8 \%), 19 \%$ Gurage $(18 \%)$. No significant difference was found between the inner and outer city age distribution, but there was a higher proportion of females in the inner city (55 vs. $52 \%$, $P=0 \cdot 0112$ ).

From 1384 HHs surveyed, 1341 (97\%) included one or more individuals of eligible age (i.e. $<50$ years), of which $1262(94 \%)$ contained at least one individual who gave a blood sample. For the $79(6 \%) \mathrm{HHs}$ who refused to give blood, there was no significant difference $(P>0.05)$ in inner:outer city distribution or distribution by age, sex, ethnicity, educational category, or migrant status, of heads of household, compared with respondent households, but a significantly lower number of persons per household in the non-respondent households $(P=0 \cdot 01)$. Of the 7735 individuals aged $<50$ years in the 1341 eligible $\mathrm{HHs}$, $4763(62 \%)$ provided a blood sample. A higher proportion of females gave blood $(2852 / 4195,68 \%$ females, vs. $1911 / 3540,54 \%$ males, $P<0 \cdot 001)$. There was no marked difference by age in the proportion of females giving blood, however, for males, the proportion declined from $74 \%$ in $0-4$ year olds, to $63 \%$ in 5-14 year olds, to $47 \%$ in adults $15-49$ years of age. The distribution by age of this sub-sample providing a blood specimen follows closely the pattern in the 1994 census and the total registered sample, although with an under-representation of adult males (male:female ratio for adults 15-49 years in the sample is $21 \cdot 2: 40 \cdot 0 \%$ and the census is $31 \cdot 3: 34 \cdot 2 \%$ [16].

For 109 out of the 4763 individuals aged $0-49$ years who gave blood, there was insufficient serum residue for measles antibody determination. We present results of analyses of measles serological data on 4654 individuals aged $<50$ years ( 1805 aged $<15$ years) arising from 1257 households in 40 kebeles.

\section{Age-prevalence of measles antibodies}

Over all ages, the estimated prevalence of seronegatives (i.e. with antibody levels $<100 \mathrm{IU} / 1$ ), with no account taken of sampling structure (i.e. assuming simple random sampling), was $4 \cdot 1 \%$. Adjusting for the survey design the estimate was little changed at 


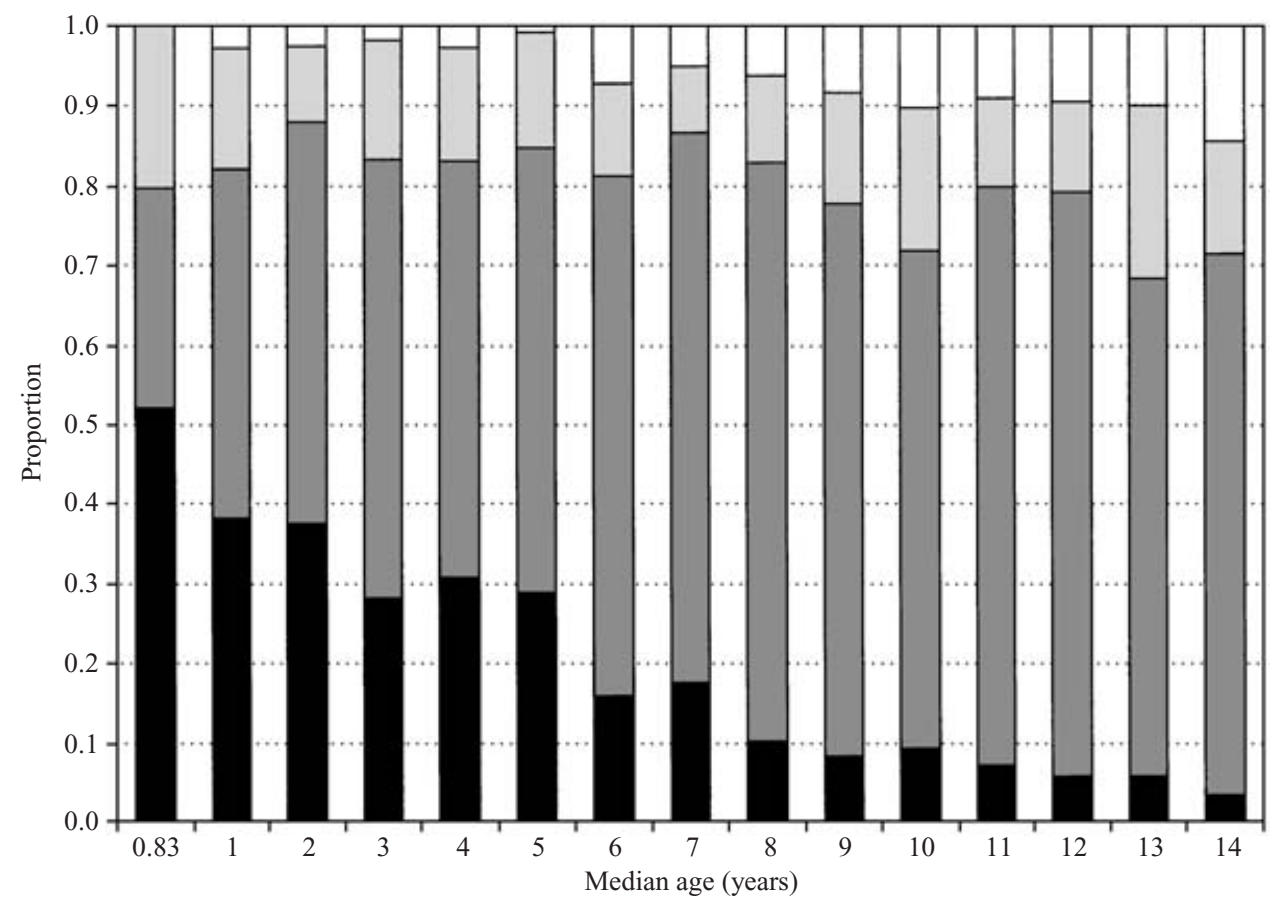

Fig. 2. Distribution of measles vaccine status in Addis Ababa, 1994, in children aged 9-11 months to 14 years, categorized as (a) vaccination verified by card (dark grey, $15 \%, n=274$ ), (b) verbal history of vaccination (medium grey, 64\%, $n=1114$ ), (c) unvaccinated (light grey, $14 \%, n=246$ ) and $(d)$ unknown (white, $7 \%, n=134$ ). Sample sizes corresponding to the age group 9-11 months and yearly age groups 1-24 years are as follows: 22, 69, 69, 100, 123, 107, 90, 141, 125, 144, 175, 114, 178, 155,156 (33 were aged $0-8$ months, and vaccine data were missing for 4 others.)

$4 \cdot 0 \%(95 \%$ CI $3 \cdot 4-4 \cdot 7)$. We estimate a design effect (ratio of variances for cluster and random sampling) of $1 \cdot 19$. Figure 1 shows the estimated age-stratified prevalence of different levels of measles-specific antibody in the population. Seronegative prevalence decreased from $66 \%(95 \%$ CI 46-82) in infants less than 9 months of age $(n=33)$, to $20 \cdot 4 \%(16 \cdot 3-25 \cdot 1)$ in 9-59 month olds, $4 \cdot 9 \%(3 \cdot 6-6 \cdot 6)$ in $5-14$ years olds, and $0 \cdot 7 \%(0 \cdot 4-1 \cdot 2)$ in adults (15-49 years). The corresponding estimated proportions of the population with low level antibody (100-255 IU/1) were $11 \cdot 2 \%$ $(4 \cdot 2-26 \cdot 4), 13 \cdot 3 \%(11 \cdot 1-15 \cdot 9), 6 \cdot 6 \%(5 \cdot 3-8 \cdot 3)$ and $1 \cdot 2 \%(0 \cdot 9-1 \cdot 6)$. The proportion of children under 15 years old who were seronegative, or had antibody levels $(\mathrm{IU} / \mathrm{l})$ of $100-255,255-500$ or $500-1000$, was $9 \cdot 3 \%(7 \cdot 0-10 \cdot 9), 8 \cdot 1 \%(7 \cdot 5-10 \cdot 4), 8 \cdot 7 \%(7 \cdot 2-10 \cdot 5)$ and $22 \cdot 9 \%(19 \cdot 0-27 \cdot 4)$, respectively. In the age classes 9-59 months and 5-14 years, the proportion of seropositives with low level antibody (100-255 IU/1) was $16 \cdot 7 \%(13 \cdot 8-20 \cdot 2)$ and $7 \cdot 0 \%(5 \cdot 5-8 \cdot 7)$, respectively. Geometric mean titre (GMT) of measles antibody in seropositives (antibody level $\geqslant 100 \mathrm{IU} / \mathrm{l}$ ) increased with age to a maximum in age class 15-19 years (Fig. 1, line). In the age group 15-49 years the antibody profile showed little change. Note that above 14 years of age very few individuals will have had measles vaccination.

\section{The reported vaccination status of children}

The distribution by age in the reported vaccination status of children aged 9 months to 14 years, i.e. 'vaccine-eligible' (VE) group, is shown in Figure 2. The age at vaccination was not recorded. Not shown are the results for 33 infants aged less than 9 months of which $29 \%$ (95\% CI 15-49) had a history of vaccination (documented by card or a verbal history), $19 \%$ by card alone. For $9-23$ months old the proportion with vaccination history was $81 \%$ (95\% CI 71-89) or with card alone was $42 \%(33-51)$. With increasing age there was a marked decline in the proportion with a card record of measles vaccination to under $10 \%$ in children aged greater than 9 years old, whereas the proportion with vaccination history was relatively stable $(79 \%$, range $68-88)$ over the age range 9 months to 14 years. The proportion whose vaccine status was unknown increased with age from $2 \%$ in 9 to 23 -month-old children to $15 \%$ in 14 year olds. Excluding these individuals of unknown status, the overall proportion of VE children with a history 
Table 1. Univariate analysis of potential factors associated with measles seronegative status $(<100 \mathrm{IU} / \mathrm{l})$ or low seropositive status (100-255 IU/l) in vaccine eligible (VE) children (9 months to 14 years of age) in Addis Ababa, 1994

\begin{tabular}{|c|c|c|c|c|c|c|}
\hline \multirow[b]{2}{*}{ Variable } & \multicolumn{3}{|c|}{ Seronegative status } & \multicolumn{3}{|c|}{ Low seropositive status* } \\
\hline & $n$ & $\%$ & $P$ & $n$ & $\%$ & $P$ \\
\hline \multicolumn{7}{|l|}{ Age (years) } \\
\hline $1-4$ & 384 & $20 \cdot 4$ & \multirow[t]{3}{*}{$0 \cdot 000$} & 305 & $16 \cdot 7$ & \multirow[t]{3}{*}{$0 \cdot 000$} \\
\hline $5-9$ & 609 & $7 \cdot 43$ & & 563 & $9 \cdot 42$ & \\
\hline $10-14$ & 779 & $2 \cdot 91$ & & 756 & $5 \cdot 15$ & \\
\hline \multicolumn{7}{|l|}{ Sex } \\
\hline M & 828 & $9 \cdot 34$ & \multirow[t]{2}{*}{$0 \cdot 116$} & 760 & $11 \cdot 6$ & \multirow[t]{2}{*}{$0 \cdot 197$} \\
\hline $\mathrm{F}$ & 934 & $7 \cdot 2$ & & 864 & $9 \cdot 91$ & \\
\hline \multicolumn{7}{|l|}{ History of measles } \\
\hline Yes & 779 & $2 \cdot 56$ & \multirow{3}{*}{$0 \cdot 000$} & 759 & $5 \cdot 32$ & \multirow[t]{3}{*}{$0 \cdot 000$} \\
\hline No & 786 & $12 \cdot 3$ & & 688 & $13 \cdot 3$ & \\
\hline Unknown & 206 & $14 \cdot 1$ & & 176 & $5 \cdot 82$ & \\
\hline \multicolumn{7}{|l|}{ Vaccination status } \\
\hline Vaccine/card & 274 & $7 \cdot 75$ & \multirow[t]{4}{*}{$0 \cdot 0485$} & 252 & $13 \cdot 8$ & \multirow[t]{4}{*}{$0 \cdot 000$} \\
\hline Vaccine/history & 1114 & $6 \cdot 57$ & & 1042 & $9 \cdot 27$ & \\
\hline Unvaccinated & 246 & $15 \cdot 4$ & & 207 & $3 \cdot 56$ & \\
\hline Unknown & 134 & $10 \cdot 1$ & & 119 & $2 \cdot 42$ & \\
\hline \multicolumn{7}{|l|}{ Stratum $\dagger$} \\
\hline Inner & 882 & $6 \cdot 88$ & \multirow[t]{2}{*}{$0 \cdot 063$} & 821 & $5 \cdot 45$ & \multirow[t]{2}{*}{$0 \cdot 000$} \\
\hline Outer & 890 & $9 \cdot 90$ & & 801 & $13 \cdot 1$ & \\
\hline \multicolumn{7}{|l|}{ Length of residence } \\
\hline All life & 1538 & $7 \cdot 52$ & \multirow[t]{2}{*}{$0 \cdot 031$} & 1420 & $9 \cdot 40$ & \multirow[t]{2}{*}{$0 \cdot 016$} \\
\hline Migrant & 234 & $12 \cdot 76$ & & 204 & $4 \cdot 41$ & \\
\hline Ethnic group & & & & & & \\
\hline Amhara & 825 & $7 \cdot 84$ & $0 \cdot 681$ & 758 & $8 \cdot 74$ & $0 \cdot 183$ \\
\hline Oromo & 299 & $10 \cdot 9$ & & 266 & $12 \cdot 3$ & \\
\hline Tigre & 167 & $6 \cdot 56$ & & 156 & $6 \cdot 39$ & \\
\hline Gurage & 364 & $8 \cdot 08$ & & 335 & $6 \cdot 87$ & \\
\hline Other & 111 & $7 \cdot 19$ & & 103 & $10 \cdot 7$ & \\
\hline Household size & & & & & & \\
\hline$<5$ & 171 & $10 \cdot 2$ & $0 \cdot 233$ & 150 & $7 \cdot 30$ & $0 \cdot 800$ \\
\hline $5-9$ & 1075 & $8 \cdot 71$ & & 980 & $9 \cdot 14$ & \\
\hline $10+$ & 526 & $6 \cdot 47$ & & 492 & $8 \cdot 52$ & \\
\hline Number sleeping in same ro & & & & & & \\
\hline$\leqslant 2$ & 219 & $7 \cdot 54$ & $0 \cdot 815$ & 202 & $7 \cdot 46$ & $0 \cdot 473$ \\
\hline $3-5$ & 943 & $8 \cdot 65$ & & 859 & $9 \cdot 75$ & \\
\hline$\geqslant 6$ & 608 & $7 \cdot 80$ & & 561 & $7 \cdot 61$ & \\
\hline Education & & & & & & \\
\hline None & 269 & $11 \cdot 4$ & $0 \cdot 371$ & 238 & $9 \cdot 07$ & 0.725 \\
\hline Read and write & 494 & $6 \cdot 67$ & & 460 & $8 \cdot 92$ & \\
\hline $1-6$ years & 399 & $7 \cdot 26$ & & 369 & $7 \cdot 04$ & \\
\hline $7-12$ years & 427 & $8 \cdot 83$ & & 389 & $8 \cdot 92$ & \\
\hline$>12$ years & 183 & $7 \cdot 99$ & & 168 & $11 \cdot 5$ & \\
\hline Ownership of house & & & & & & \\
\hline Rent/other & 1091 & $7 \cdot 40$ & $0 \cdot 178$ & 1007 & $7 \cdot 62$ & $0 \cdot 044$ \\
\hline Own & 670 & $9 \cdot 67$ & & 607 & $10 \cdot 8$ & \\
\hline Type of wall & & & & & & \\
\hline Wood, bamboo, canvass & 1604 & $8 \cdot 42$ & $0 \cdot 341$ & 1467 & $8 \cdot 30$ & $0 \cdot 060$ \\
\hline Bricks/stone & 163 & $6 \cdot 18$ & & 152 & $13 \cdot 6$ & \\
\hline
\end{tabular}


Table 1. (cont.)

\begin{tabular}{|c|c|c|c|c|c|c|}
\hline \multirow[b]{2}{*}{ Variable } & \multicolumn{3}{|c|}{ Seronegative status } & \multicolumn{3}{|c|}{ Low seropositive status* } \\
\hline & $n$ & $\%$ & $P$ & $n$ & $\%$ & $P$ \\
\hline \multicolumn{7}{|l|}{ Type of roof } \\
\hline Corregated iron & 1728 & $8 \cdot 20$ & 0.691 & 1584 & $8 \cdot 67$ & $0 \cdot 343$ \\
\hline Stone, concrete, tile & 39 & $9 \cdot 69$ & & 35 & $12 \cdot 4$ & \\
\hline \multicolumn{7}{|l|}{ Number of rooms§ } \\
\hline 1 & 390 & $10 \cdot 4$ & $0 \cdot 284$ & 347 & $6 \cdot 40$ & $0 \cdot 181$ \\
\hline 2 & 672 & $7 \cdot 57$ & & 620 & $8 \cdot 63$ & \\
\hline $3+$ & 704 & $7 \cdot 61$ & & 651 & $10 \cdot 2$ & \\
\hline \multicolumn{7}{|l|}{ Number of beds } \\
\hline $0-1$ & 386 & $9 \cdot 54$ & $0 \cdot 526$ & 348 & $7 \cdot 14$ & $0 \cdot 108$ \\
\hline 2 & 531 & $8 \cdot 15$ & & 486 & $7 \cdot 09$ & \\
\hline $3+$ & 847 & $7 \cdot 61$ & & 783 & $10 \cdot 5$ & \\
\hline \multicolumn{7}{|l|}{ Electricity supply } \\
\hline Private meter & 1169 & $8 \cdot 33$ & $0 \cdot 782$ & 1071 & $8 \cdot 46$ & 0.685 \\
\hline Other & 595 & $7 \cdot 94$ & & 546 & $9 \cdot 20$ & \\
\hline \multicolumn{7}{|l|}{ Ownership of radio } \\
\hline No & 314 & $10 \cdot 8$ & $0 \cdot 077$ & 279 & $10 \cdot 2$ & $0 \cdot 379$ \\
\hline Yes & 1450 & $7 \cdot 64$ & & 1338 & $8 \cdot 41$ & \\
\hline \multicolumn{7}{|l|}{ Source of water } \\
\hline Public source & 268 & $6 \cdot 77$ & 0.655 & 249 & $10 \cdot 8$ & 0.239 \\
\hline Shared tap & 822 & $8 \cdot 53$ & & 750 & $7 \cdot 23$ & \\
\hline Own tap & 674 & $8 \cdot 34$ & & 618 & $9 \cdot 84$ & \\
\hline
\end{tabular}

* Analysis excludes seronegatives.

$\dagger$ Stratum of city; inner high density $\left(37235 / \mathrm{km}^{2}\right)$ and outer lower density $\left(10803 / \mathrm{km}^{2}\right)$.

$\$$ Migrant is a person born outside Addis Ababa, living in the city or a visitor at the time of the survey.

$\S$ Number of rooms in a household does not include kitchen or store rooms.

of vaccination was $85 \%(95 \%$ CI $82-88)$ and did not differ by age $(P=0 \cdot 1404)$. No difference was observed between inner and outer city in vaccination prevalence, and in all clusters exceeded $65 \%$. A significantly higher fraction of lifelong residents of Addis Ababa had a history of vaccination relative to migrants (not born in Addis Ababa) (86 vs. 33\%, $P<0 \cdot 0001)$.

\section{Vaccine failure rate and failure of vaccination}

Of children aged 9-23 months with history of measles vaccination $21 \%$ (95\% CI 12-34) were seronegative (with no difference in seronegative prevalence between those with cards or verbal history). This result is unaffected if we exclude migrants. In the total VE group ( 9 months to 14 years) $8.4 \%$ were identified as seronegative (Fig. 1), of which $14 \cdot 3 \%(9 \cdot 4-21 \cdot 3)$ had card evidence, and 50.8\% (39.3-62.3) verbal history, of measles vaccination. In seronegatives aged 9-23 months $41 \%$ (18-69) had card evidence and $42 \%$ (18-69) had verbal history of vaccination.

\section{Relationship between history of measles, measles vaccination and seroprevalence}

For each person we recorded his/her history of measles-like illness (definitions: yes, no, did not know), the condition well known locally as Kufugn. The proportion with a reported measles-like episode was $43 \%$ in children under 15 years of age and $55 \%$ of adults 15-49 years old, and did not differ between city strata. In the VE group the proportion with a history of measles was marginally lower in those with card evidence of vaccination $(33 \%)$ than those reportedly unvaccinated $(43 \%)(P=0 \cdot 116)$.

The reliability of a reported history of measles was assessed in 212 individuals of the VE group who were reported as unvaccinated (here we are assuming reported absence of vaccination is reliable). We excluded individuals with unknown history of measles. Of 182 measles seropositive individuals 103 reported a measles history (sensitivity of $57 \%$ ), and 27 out of 30 seronegatives had no history of measles (specificity of $90 \%$ ). Thus we had few false positive reports of 
Table 2. Multiple logistic regression models of potential factors associated with measles seronegative status $(<100 \mathrm{IU} / \mathrm{l})$ or low seropositive status (100-255 IU/l) in VE children in Addis Ababa, 1994

\begin{tabular}{|c|c|c|c|c|c|c|}
\hline \multirow[b]{2}{*}{ Variable } & \multicolumn{3}{|c|}{ Seronegative status } & \multicolumn{3}{|c|}{ Low positive status* } \\
\hline & Adj. OR & $95 \% \mathrm{CI}$ & $P$ & Adj. OR & $95 \% \mathrm{CI}$ & $P$ \\
\hline \multicolumn{7}{|l|}{ Age (years) } \\
\hline $1-4$ & $1 \cdot 00$ & & & $1 \cdot 00$ & & \\
\hline $5-9$ & $0 \cdot 31$ & $0 \cdot 20-0 \cdot 48$ & $0 \cdot 000$ & $0 \cdot 58$ & $0 \cdot 37-0 \cdot 91$ & $0 \cdot 019$ \\
\hline $10-14$ & $0 \cdot 10$ & $0 \cdot 05-0 \cdot 20$ & $0 \cdot 000$ & $0 \cdot 37$ & $0 \cdot 22-0 \cdot 63$ & $0 \cdot 000$ \\
\hline \multicolumn{7}{|l|}{ History of measles } \\
\hline Yes & $1 \cdot 00$ & & & $1 \cdot 00$ & & \\
\hline No & $3 \cdot 82$ & $2 \cdot 29-6 \cdot 39$ & $0 \cdot 000$ & $2 \cdot 29$ & $1 \cdot 55-3 \cdot 37$ & $0 \cdot 000$ \\
\hline Unknown & $6 \cdot 88$ & $3 \cdot 84-11 \cdot 38$ & $0 \cdot 000$ & $2 \cdot 07$ & $0 \cdot 98-4 \cdot 36$ & $0 \cdot 056$ \\
\hline \multicolumn{7}{|l|}{ Vaccination status } \\
\hline Vaccine/card & $1 \cdot 00$ & & & $1 \cdot 00$ & & \\
\hline Vaccine/history & $1 \cdot 82$ & $1 \cdot 08-3 \cdot 07$ & $0 \cdot 025$ & $1 \cdot 01$ & $0 \cdot 63-1 \cdot 62$ & 0.973 \\
\hline Unvaccinated & $4 \cdot 30$ & $1 \cdot 96-9 \cdot 43$ & $0 \cdot 001$ & $0 \cdot 35$ & $0 \cdot 17-0 \cdot 71$ & $0 \cdot 005$ \\
\hline Unknown & $2 \cdot 20$ & $0 \cdot 94-5 \cdot 15$ & $0 \cdot 069$ & $0 \cdot 23$ & $0 \cdot 07-0 \cdot 80$ & $0 \cdot 022$ \\
\hline \multicolumn{7}{|l|}{ Stratum } \\
\hline Inner & ni† & & & $1 \cdot 00$ & & \\
\hline Outer & & & & $2 \cdot 68$ & $1 \cdot 84-3 \cdot 91$ & $0 \cdot 000$ \\
\hline
\end{tabular}

* Analysis excludes seronegatives.

$\dagger$ ni, not included in model.

measles, but a high proportion of individuals failed to recognize or remember they had measles. The predictive value for a negative history of measles is estimated to be only $25 \%(27 / 106)$. Furthermore, we find that of $1521 \mathrm{VE}$ individuals reporting a history of measles illness, vaccination or both, 97 (6\%) were seronegative. Thus of the total of 148 seronegative children in this age range, $66 \%$ would not have been identified as susceptible, suggesting that historical information is unreliable in identifying at-risk individuals.

\section{Risk factors for measles seronegativity in $\mathrm{VE}$ children}

In children aged 9 months to 14 years, absences of a history of vaccination, or of a measles-like illness, were strong and independent predictors of seronegative status (Tables 1, 2, Fig. $3 a$ ). Unvaccinated individuals without a history of measles illness were roughly 10 times more likely to be seronegative than those vaccinated and who reported measles illness $(25.6 \%$; $95 \%$ CI $14 \cdot 8-40 \cdot 5$ vs. $2 \cdot 6 \% ; 1 \cdot 6-4 \cdot 3)$. No other independently significant associations were identified. Interactions between age and each of sex, history of measles, vaccination status, stratum and length of residence were all found to be non-significant.

\section{Risk factors for low level antibody in VE children}

In seropositive children aged 9 months to 14 years a history of vaccination and absence of measles-like illness were strongly associated with high prevalence of low-level antibody status (Tables 1, 2, Fig. 3b) and with low GMT (Fig. 3c). We observed a 14-fold higher prevalence of low positives in vaccinated individuals without a history of measles illness $(14.6 \% ; 95 \%$ CI $11.9-17 \cdot 7)$ than in individuals unvaccinated but with measles-like illness $(1 \cdot 0 \% ; 95 \%$ CI $0 \cdot 1-7 \cdot 5)$.

Measles illness history was available for the full age range. The GMT measles antibody in seropositive individuals with a history of symptomatic measles remained roughly constant throughout the age range at a level always higher than in those who reported no measles history, other than age group 30-34 years. In those without measles history there was a trend for increase in GMT throughout childhood and a trend for decrease in later life from 35-39 to 45-49 years.

Significantly higher prevalence of low-level antibody was found in the outer city than the inner city (13.2 and $5.44 \%$, respectively) (Table 2). These results cannot be attributed to differences in vaccine uptake or in history of measles illness. No other independent risk factors were identified and no significant interactions were identified with age. 

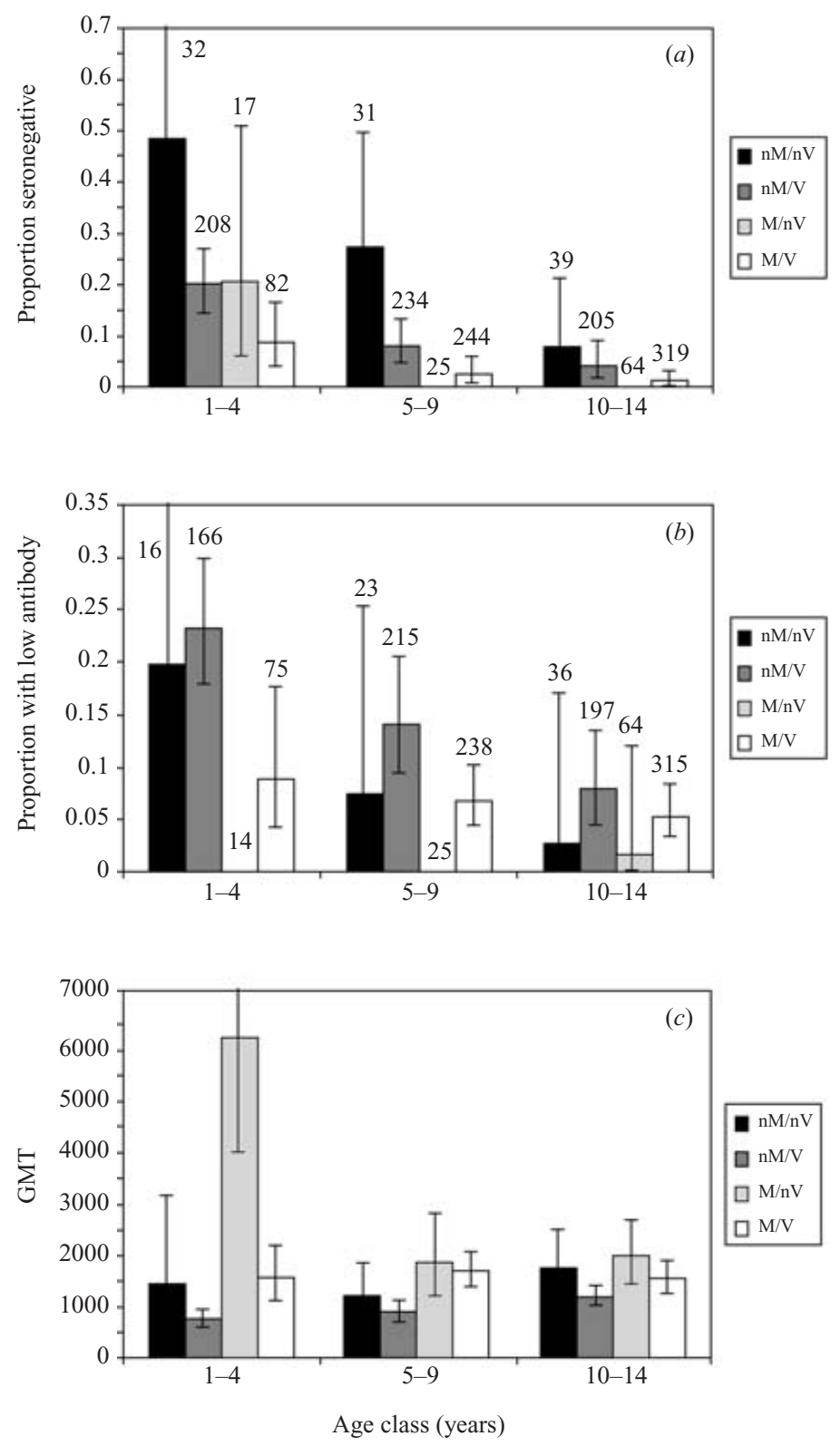

Fig. 3. Age-specific proportions $(a)$ seronegative $(<100 \mathrm{IU} / 1)$, or $(b)$ with low level antibody (100-255 IU/1, panel (b) seropositives only), and $(c)$ GMT measles antibody by age (seropositives only), stratified by history of measles vaccination (card or verbal) and illness. Dark bars: no illness, no vaccine (nMnV); dark grey bars: no illness, vaccine (nMV); light grey bars: illness, no vaccine (MnV); open bars: illness, vaccine (MV). Bars show $95 \%$ confidence limits, and indicated sample sizes exclude individuals with an unknown history of vaccine or illness [sample sizes for panels $(b)$ and $(c)$ are equal].

\section{Measles incidence}

Assuming a constant force of infection, Figure 4 shows the fit of the catalytic infection model [eq. (1)] to the observed proportions susceptible in ages 1-14 years, inclusive (open markers) or exclusive (filled markers) of individuals with migrant status $(n=233)$. The force of infection $\lambda$ when migrants were excluded was estimated to be $23 \%$ /year (95\% CI $21-26 \%$ ), slightly higher than the value of $21 \%$ /year (19-24\%) inclusive of migrants. The effect on the estimated force of infection of using a reduced age range was small (range in estimated $\lambda$ was $22-27 \%$ ) and there was no significant improvement in model fit allowing a non-constant force of infection (with 2 or 3 age groups). These results suggest that our estimate of the force of infection in Addis 1994 of $23 \%$ is robust to the main assumptions of time dependence both in vaccine coverage and measles incidence. Given that the proportion susceptible, $x$, in those aged less than 


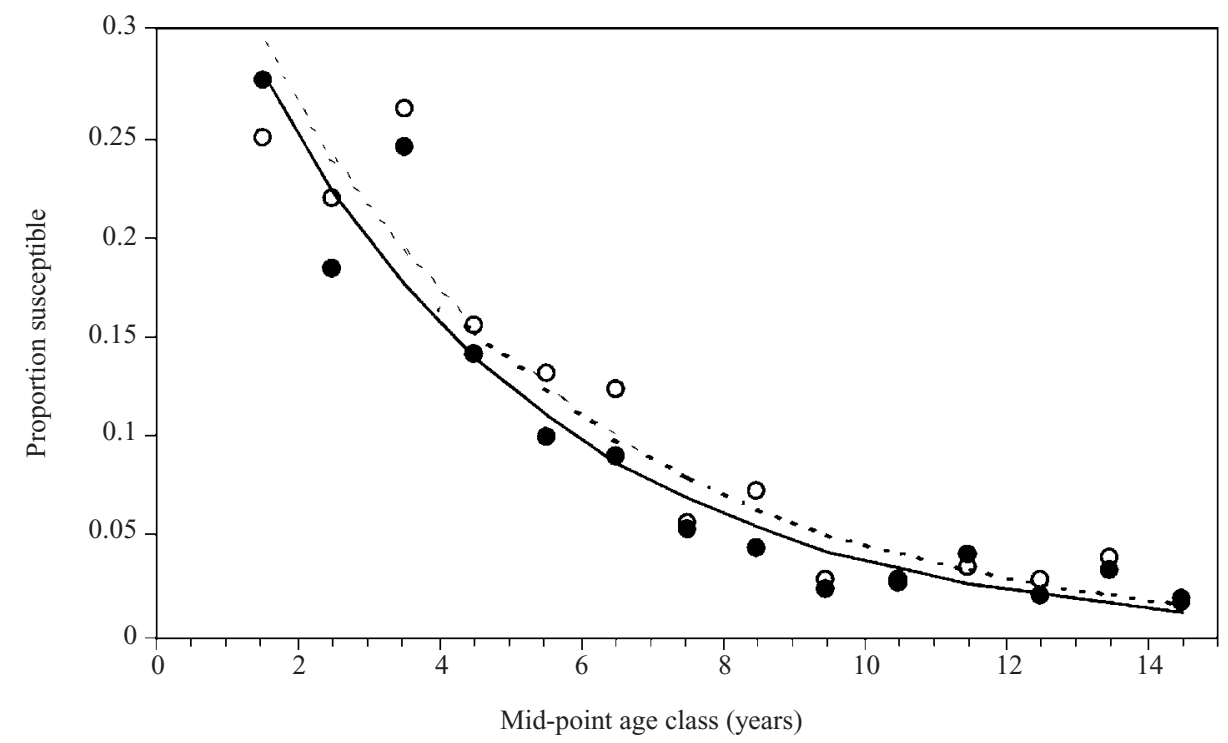

Fig. 4. Age-specific proportions seronegative in Addis Ababa 1994 with catalytic infection model fit that assumes a constant force of infection, $\lambda$ (per person incidence rate). Shown are the observed and predicted prevalence for total inhabitants (open markers, dotted line) and for lifelong inhabitants only (filled markers, solid line). Estimates: all inhabitants $\lambda=0 \cdot 215(95 \%$ CI $0 \cdot 192-0 \cdot 239), x(0)=0 \cdot 402$; excluding migrants $\lambda=0 \cdot 234(95 \%$ CI $0 \cdot 207-0 \cdot 262), x(0)=0 \cdot 395$.

15 years is $9 \%$ and in those aged $<5$ years is $24 \%$, then the attack rate (i.e. $\lambda . x .1000)$ for each age class is estimated at $21 / 1000$ and 55/1000 per year, respectively.

\section{DISCUSSION}

A large cross-sectional seroprevalence survey was conducted in order to characterize measles epidemiology and immunity in the population of Addis Ababa, Ethiopia. The study employed a stratified clusterbased household sample through which has been generated precise and detailed seroepidemiological information on measles in a developing country urban setting experiencing moderately high measles vaccine coverage. The sample was representative, insofar as the socio-demographic structure of the sample holds remarkably well with that recorded in the 1994 census of Addis Ababa [13, 15]. There are few previous reports of antibody prevalence from such large and representative sample surveys [3, 4] and particularly for Africa.

The ELISA kit used to quantify measles specific antibody levels has been found to compare well against other commercially available ELISAs and to be suited to quantitative measurement [21]. Reporting of quantitative antibody measurement in standard international units is important for comparison between studies, although the use of different assay formats (e.g. ELISA, haemagglutination inhibtion, and antibody neutralization) in different studies argues for caution in this process. The threshold for seronegativity of $100 \mathrm{IU} / 1$ is similar to some previous, reports $[3,4,10,21]$, though not all [7]. The cut-off level for low positives of $255 \mathrm{IU} / 1$ follows that previously reported [4]. Levels of 500 and 1000 have been suggested as defining the cut-off for protection against infection and clinical measles [9]. There is considerable variation within the literature on where these boundaries lie and on the relationship between specific-antibody levels and protection from infection and disease. The material has been reviewed elsewhere $[4,8-10]$, and further interpretation is beyond the scope of this paper.

Based mostly on verbal history (Fig. 2) our results indicate that measles vaccine coverage has been moderately high and relatively stable at about $80 \%$ in the population of Addis Ababa for over a decade. This is consistent with the available official records. However, we obtain a minimum estimate for primary vaccine failure rate of $21 \%(95 \%$ CI 12-34) from seronegative children 9-23 months old with vaccination history (some of these children may have antibody induced by natural infection). This suggests a potential problem in the effectiveness in delivery of measles vaccine in Addis Ababa. Higher coverage of lifelong resident children compared to migrants reflects known differences between urban and rural Ethiopia [26]. 
Highest proportions seronegative $(<100 \mathrm{IU} / \mathrm{l})$ were identified in infants less than 9 months $(66 \%)$ and children aged $1-3$ years $(25 \%)$ and $4-6$ years $(13 \%)$. In ages 7 years and upwards the proportion with detectable antibody was in excess of $93 \%$, rising to $99 \%$ in adults 15-49. Statistics for Addis Ababa suggest a population under demographic transition; the birth rate has markedly declined over the past 30 years [13]. In 1994, $0-4$ year olds represented only $8 \%$ of the total population and the largest proportion (16\%) was in the age group 15-19 years [16]. Given this age structure there is in fact a fairly even distribution of numbers of susceptibles throughout the child age range. Analysis of the change with age in proportion seronegative using a catalytic infection model yields a most likely estimate of measles incidence rate of around 22-23 per 100 susceptibles per annum in children 1-14 years of age, indicative of continued high measles transmission. This is despite moderately high measles vaccination levels. Unfortunately there are no reliable reported statistics for measles notifications in Ethiopia. Measles seronegativity was strongly and independently associated with an absence of a history of vaccination or of measles illness. Other than age, no other independent risk factors for seronegativity were identified.

The analysis of antibody levels concentrates on seropositive individuals falling into the low level category (100-255) for whom there is some evidence of susceptibility to either mild clinical or sub-clinical reinfection $[8,9]$. A significant proportion $(8 \%)$ of children (aged $<15$ years) were identified as having low-level antibody (100-255 IU/1). Stratified analysis of seropositives confirms a strong independent association between a history of measles vaccination and low measles antibody level. The observed decline in the proportion with low level antibody over the childhood age range is predominantly associated with an increase in GMT in vaccinated seropositives, and may arise from the boosting of low-level antibody by exposure to continued circulating measles virus. Such a decrease in the prevalence of low-level seropositivity was not observed in a study of children in Bolivia, perhaps reflecting more effective control of measles virus in the South American population [3]. Absence of a history of measles illness was also strongly associated with low-level seropositivity. An association between measles history and high antibody level was previously reported for children in a study in Bolivia [3]. Our study identified a strong independent association between low level antibody and residence in the outer less densely populated region of the city. It is possible that this is the result of less boosting of antibodies by exposure to measles, associated with lower population density.

We attach little importance directly to the estimated proportion with low antibody level of between 100-255 IU/1. Instead, we highlight that this result adds to the reports of high proportions of individuals with low positive antibody levels in vaccinated populations $[3,4,8]$, for whom a role in continued transmission of measles, though unlikely, cannot be discounted [8]. The inference from this and other studies $[8,27]$ is that higher levels of vaccination (single dose) and an accompanying decrease in the boosting effect of viral exposure are likely to further increase the proportion with low-level antibody. An emerging population of vaccinated low positive individuals may be an issue in considering the merits of supplementary doses of measles vaccine to boost immunity.

In late 1998 mass measles campaigns were conducted for the first time in Ethiopia in nine selected urban cities, including Addis Ababa, targeting children aged 9-59 months old, as part of the accelerated measles control strategy in Ethiopia [18]. As is usual in campaigns, measles vaccine was offered irrespective of past measles vaccination or illness. The findings of the current study, as previously [3], show that historical information on measles vaccination and illness is of little use in identifying seronegatives, and would not support a more targeted approach to campaign vaccination. Our survey in 1994 indicates $20 \%$ seronegative and $17 \%$ low seropositive in 9- to 59-month-old children. Together with evidence for continued high rate of transmission in Addis (despite moderately high level vaccination), these data justify the need for the accelerated control effort implemented in Addis Ababa. However, although the survey indicated only $5 \%$ seronegative and $7 \%$ low positive in the 5-14 years age range, this age group comprises $24 \%$ of the total population compared to only $8 \%$ in those $<5$ years. Targeting children under 5 is appropriate when the aim is to reduce measlesassociated mortality. Inclusion of older children in campaigns, as in other countries [28], could have a greater impact on reducing the numbers of susceptibles, and consequently of cases, and would be necessary if the immunization programme aimed to interrupt transmission [29]. Our conclusion is that the benefits of the campaign are likely to be short lived in the absence of increased routine immunization 
coverage at age 9 months or repeated campaigns [30]. Careful consideration should now be given to the prevention of future epidemics, the like of which have been observed elsewhere following campaign intervention [31, 32]. The control of measles in Addis Ababa has wider implications for measles persistence in Ethiopia given its large size (roughly $1 / 25$ of the total population). Further antibody prevalence surveys to assess the impact of the recent campaigns would be beneficial in developing immunization policy. Oral-fluid methods have been identified as useful in this process [12, 33-35].

\section{ACKNOWLEDGEMENTS}

The study was made possible by the generous participation of Addis Ababa inhabitants and the support of the Ministry of Health, Region 14 Health Bureau, and Kebele Officials. In particular we thank Dr Eyob Tsegaye (previously Head of Region 14 Health Bureau, Addis Ababa) and Dr Wondemagegnehu Alemu (previously EPI manager, Ministry of Health, Addis Ababa) for their support in conducting the survey work. D.J.N. was funded by The Royal Society during this work. The study had financial support from the Wellcome trust (Project grant no. 039056).

\section{REFERENCES}

1. Babad HR, Nokes DJ, Gay NJ, Miller E, MorganCapner P, Anderson RM. Predicting the impact of measles vaccination in England and Wales: model validation and analysis of policy options. Epidemiol Infect 1995; 114: 319-44.

2. Gay NJ, Hesketh LM, Morgan-Capner P, Miller E. Interpretation of serological surveillance data for measles using mathematical models: implications for vaccine strategy. Epidemiol Infect 1995 ; 115: 139-56.

3. Cutts FT, Bartoloni A, Guglielmetti P, et al. Prevalence of measles antibody among children under 15 years of age in Santa Cruz, Bolivia: implications for vaccination strategies. Trans Royal Soc Trop Med Hyg 1995; 89: 119-22.

4. Cox MJ, Azevedo RS, Massad E, Fooks AR, Nokes DJ. Measles antibody levels in a vaccinated population in Brazil. Trans Royal Soc Trop Med Hyg 1998; 92: 227-30.

5. de Melker H, Pebody RG, Edmunds WJ, et al. The seroepidemiology of measles in Western Europe. Epidemiol Infect $2001 ; \mathbf{1 2 6}$ : 249-59.

6. Gay N, Ramsay M, Cohen B, et al. The epidemiology of measles in England and Wales since the 1994 vaccination campaign. CDR 1997; 7: R17-R21.
7. Janaszek W, Gut W, Gay NJ. The epidemiology of measles in Poland: prevalence of measles virus antibodies in the population. Epidemiol Infect 2000; 125: 385-92.

8. Mossong J, Nokes DJ, Edmunds WJ, Cox MJ, Ratnam S, Muller CP. Modeling the impact of subclinical measles transmission in vaccinated populations with waning immunity. Am J Epidemiol 1999; 150: 1238-49.

9. Lee MS, Nokes DJ, Hsu HM, Lu CF. Protective titres of measles neutralising antibody. J Med Virol 2000; 62: 511-7.

10. Lee MS. An investigation of measles elimination in Taiwan: seroepidemiology and modelling. Oxford: Oxford University, 1999: 106.

11. Nigatu W, Jin L, Cohen BJ, et al. Measles virus strains circulating in Ethiopia in 1998-1999: Molecular characterisation using oral fluid samples and identification of a new genotype. J Med Virol 2001; 65: 373-80.

12. Nokes DJ, Enquselassie F, Nigatu W, et al. Has oral fluid the potential to replace serum for the evaluation of population immunity levels? A study of measles, rubella and hepatitis B in rural Ethiopia. Bull WHO 2001; 79: 588-95.

13. Cutts FT, Abebe A, Messele T, et al. Sero-epidemiology of rubella in the urban population of Addis Ababa, Ethiopia. Epidemiol Infect 2000; 124: 467-79.

14. Nokes DJ, Nigatu W, Abebe A, et al. A comparison of oral fluid and serum for the detection of rubella-specific antibodies in a community study in Addis Ababa, Ethiopia. Trop Med Int Health 1998; 3: 258-67.

15. Fontanet AL, Messele T, Dejene A, et al. Age- and sex-specific HIV-1 prevalence in the urban community setting of Addis Ababa, Ethiopia. Aids 1998; 12: 315-22.

16. Population Housing Census Commission. The 1994 population and housing census of Ethiopia. Results for Addis Ababa, Vol 1 - statistical report. Addis Ababa, Ethiopia: Central Statistical Authority, 1995.

17. Family Health Department. Report of a joint national review of the expanded programme on immunization (E.P.I.). Addis Ababa: Ministry of Health, 1995.

18. Family Health Department. Supplementary activities aimed at achieving measles control in Ethiopia for 1999-2000. Addis Ababa: Family Health Department, Ministry of Health, 1999.

19. Bennett S, Woods T, Liyange WM, Smith DL. A simplified general method for cluster-sample surveys of health in developing countries. World Health Stat Q 1991; 44: 98-106.

20. Population Housing Census Commission. The 1984 population census of Ethiopia. Results for Addis Ababa. Addis Ababa: Central Statistical Authority, 1985.

21. Hesketh L, Charlett A, Farrington P, Miller E, Forsey T, Morgan-Capner P. An evaluation of nine commercial EIA Kits for the detection of measles specific IgG. J Virol Meth 1997; 66: 51-9.

22. Dean AG, Dean JA, Coulombier D, et al. Epi Info, Version 6.02: a word processing, database, and statistical program for epidemiology on microcomputers. 
Atlanta, GA, USA: Centers for Disease Control and Prevention, 1994.

23. Ades AE, Nokes DJ. Modeling age- and time-specific incidence from seroprevalence:toxoplasmosis. Am J Epidemiol 1993; 137: 1022-34.

24. Nokes DJ, Forsgren M, Gille E, Ljungstrom I. Modelling longitudinal toxoplasma seroprevelence in Stockholm, Sweden. Parasitol 1993; 107: 33-40.

25. Clayton D, Hills M. Statistical models in epidemiology. New York: Oxford University Press Inc., 1993.

26. Tesfaye F, Enquselassie F, Ali K, Andom G. EPI coverage in Adami-Tulu Woreda. Ethiop J Health Develop 1997; 11: 109-13.

27. Whittle HC, Aaby P, Samb B, Jensen H, Bennett J, Simondon F. Effect of subclinical infection on maintaining immunity against measles in vaccinated children in West Africa. Lancet 1999; 353: 98-102.

28. Dietz V, Cutts F. The use of mass campaigns in the expanded program on immunization: a review of reported advantages and disadvantages. Int $\mathbf{J}$ Health Services 1997; 27: 767-90.

29. Biellik R, Madema S, Taole A, et al. First 5 years of measles elimination in southern Africa: 1996-2000. Lancet 2002; 359: 1564-8.
30. Nokes DJ, Swinton J. Vaccination in pulses: a strategy for global eradication of measles and polio? Trends Microbiol 1997; 5: 14-9.

31. Pan American Health Organisation. Measles in the Americas, 1998. EPI Newsletter 1998; 20: 1-2.

32. Bilkis MD, Barrero PR, Mistchenko AS. Measles resurgence in Argentina: 1997-8 outbreak. Epidemiol Infect 2000; 124: 289-93.

33. Nokes DJ, Enquselassie F, Vyse A, Nigatu W, Cutts FT, Brown DW. An evaluation of oral-fluid collection devices for the determination of rubella antibody status in a rural Ethiopian community. Trans R Soc Trop Med Hyg 1998; 92 : 679-85.

34. Nigatu W, Nokes DJ, Enquselassie F, et al. Detection of measles specific $\mathrm{IgG}$ in oral fluid using an FITC/anti-FITC IgG capture enzyme linked immunosorbent assay (GACELISA). J Virol Meth 1999; 83: $135-44$.

35. Vyse AJ, Brown DW, Cohen BJ, Samuel R, Nokes DJ. Detection of rubella virus-specific immunoglobulin $G$ in saliva by an amplification-based enzyme-linked immunosorbent assay using monoclonal antibody to fluorescein isothiocyanate. J Clin Microbiol 1999; 37: $391-5$. 